\title{
UMA ANÁLISE DA RELAÇÃO ENTRE VALORES PESSOAIS E SATISFAÇÃÓ COM O TRABALHO
}

\author{
Data de submissão: $18 / 02 / 2015$ \\ Aceite: 14/02/2017 \\ Diana Abreu von Borell ${ }^{1}$ \\ Claudio Eduardo Ramos Camfield ${ }^{2}$ \\ Ricardo Gouveia Rodrigues ${ }^{3}$
}

\section{RESUMO}

O objetivo deste estudo foi analisar a relação entre os valores pessoais e a satisfação com o trabalho. Para isso, foi feita uma regressão linear múltipla, cujas variáveis independentes foram valores pessoais e a variável dependente a satisfação com o trabalho. Os dados para este ensaio foram coletados a partir da base de dados European Social Survey com uma amostra válida de 820 indivíduos portugueses, quem participaram de um inquérito social o qual é aplicado anualmente em 20 países europeus aproximadamente. Das 4 hipóteses formuladas no estudo, 3 foram rejeitadas, revelando que os valores pessoais de autotranscedência e conservação não possuem relação positiva com a satisfação com o trabalho, assim como o valor de autopromoção não possui uma relação negativa e significante, como sugerido. $O$ valor de abertura à mudança, entretanto, apresentou uma relação negativa e significante ao nível de 5\%, porém fraca. Os resultados sugeriram que os valores pessoais não possuíram poder explicativo relevante, sobre a satisfação com o trabalho, na presente amostra. Portanto, sugere-se a inclusão de outras variáveis no modelo para fornecer melhor contribuição com a literatura sobre o tema, assim como para gestores os quais considerem relevante a satisfação do funcionário com o seu trabalho.

Palavras-chave: Satisfação com o Trabalho. Valores Pessoais.

1 É graduada em Nutrição pela Universidade Católica Salesiana do Espírito Santo - Vitória, UNISALES, mestrado em Administração de Empresas pelo Instituto Capixaba de Pesquisa em Contabilidade, Economia e Finanças, FUCAPE e PhD em Gestão com ênfase em Marketing pela Universidade da Beira Interior, UBI, Portugal. Covilhã, - Portugal. E-mail: daiaborell@yahoo.com.br

2 É professor na Universidade Federal de Santa Maria, UFSM - Campus Palmeira das Missões - Brasil, doutor em gestão pela Universidade da Beira Interior - Portugal e membro do CEFAGE-UBI Research Center, Portugal. Palmeira das Missões - RS. Brasil E-mail: claudiocamfield@hotmail.com

3 É Professor na Universidade da Beira Interior, e vice-diretor do centro de investigação NECE-UBI. Tem um doutoramento em Gestão. Os seus principais interesses de investigação são o empreendedorismo e o Marketing. Tem mais de 40 publicações científicas revistas por pares, participou em vários projetos de investigação, e coordenou um projeto internacional versando o impacto da Educação para o Empreendedorismo. Covilhã, - Portugal. E-mail: rgrodrigues@ubi.pt 


\section{INTRODUÇÃO}

No decorrer dos anos, percebem-se muitos estudos (EALIAS; JORGE, 2012; TAMAYO, 2001; PORTER et. al, 1974) sobre a satisfação no trabalho, com abordagens e resultados diversos, este é um tema importante devido ao seu impacto sobre o comportamento dos indivíduos no ambiente de trabalho (TAMAYO, 2001; ZALEWSKA, 1999). Hackman e Oldman (1980) sustentaram que a satisfação no trabalho é uma das áreas mais estudadas no campo de Recursos Humanos, visto que a satisfação das pessoas no trabalho possui uma relação direta com a produtividade, o absentismo e a rotatividade.

Diversos teóricos buscaram conceituar a satisfação no trabalho, sem que houvesse um senso comum (GEORGE; JONES, 1999; SEAL; KNIGHT, 1988; BRIEF, 1988; LOCKE, 1976), levando a crer que a satisfação no trabalho possui uma natureza relativa. No entanto, esses autores mostram em comum a visão de que a satisfação no trabalho é explicada tanto por fatores contextuais, como relação com líderes, cultura organizacional ou posição hierárquica, como por características individuais.

Dentre as características de indivíduos que podem afetar a satisfação no trabalho, nota-se a opção de estudar a relação entre a satisfação no trabalho e os valores humanos, uma vez que estes são tratados na literatura como antecedentes explicativos do comportamento humano (HASSAN, 2011; VILAS BOAS; SETTE; BRITO, 2006; CHRYSSOHOIDIS; KRYSTALLIS, 2005; VERLANKEN; HOLLAND, 2002; GRUNERT; JUHL, 1995).

A abordagem de valores escolhida para este estudo defende que valores são crenças que orientam os indivíduos não só em suas escolhas e ações, mas também no julgamento que eles fazem de situações (SCHWARTZ, 1994). Como a satisfação no trabalho envolve sentimentos e crenças (BRIEF, 1988), este estudo trouxe como problema de pesquisa a seguinte pergunta: de que forma os valores pessoais podem interferir na satisfação humana com o trabalho? Para responder a esta questão, este estudo objetivou analisar a relação entre os valores pessoais e a satisfação com o trabalho.

Por se tratar de um tema ainda atual o qual recebe muita atenção principalmente por parte dos investigadores das áreas das Ciências Sociais e Humanas, este estudo espera proporcionar uma contribuição por meio de uma investigação quantitativa com uma amostra da população portuguesa, no qual buscou-se inovar no sentido de identificar os valores de um indivíduo e verificar de que forma esses interferem na satisfação para as suas atividades laborais.

Visando estruturar de uma maneira simples e intuitiva, este artigo contempla na seção seguinte a contextualização teórica pertinente aos temas, iniciando pela temática da satisfação no trabalho e por conseguinte a teoria dos valores pessoais. Em seguida são apresentadas as hipóteses de investigação. Na terceira seção demonstram-se os aspectos metodológicos utilizados no estudo, incluindo a explicação e a apresentação do modelo estudado na análise da Regressão Múltipla. Na sequência (quarta seção) são apresentados e discutidos os resultados advindos da análise estatística e por fim, na última seção são colocadas as considerações finais. 


\section{REVISÃO DE LITERATURA}

\subsection{Satisfação no trabalho}

O tema sobre satisfação no trabalho é complexo e no decorrer dos anos veio recebendo várias definições, pois se encontra em um nível de subjetividade abrangente, podendo variar entre pessoas em um determinado grupo, assim como pode sofrer uma variabilidade ao longo do tempo, conforme determinadas circunstâncias vão ocorrendo (TAMAYO, 2001).

Na literatura são encontradas diversas abordagens para a satisfação no trabalho, entretanto um dos primeiros que se propôs a investigar o assunto foi Hoppock (1935), quem considerou que a satisfação no trabalho é uma manifestação sentimental do ser humano no ambiente de trabalho e que poderia estar ligada ao campo tanto físico como psicológico. Posteriormente, muitos outros investigadores buscaram se aprofundar sobre a temática, conforme podem ser observados alguns desses a seguir.

Vroom (1964, p. 99), usou os termos "satisfação no trabalho" e "atitudes no trabalho" como sinônimos, e definiu satisfação no trabalho como "percepções afetivas por parte dos indivíduos em resultado do trabalho que realizam". Corroborando a uma emoção positiva de bem-estar, Locke (1969) definiu satisfação no trabalho como o resultado da avaliação que o trabalhador tem sobre o seu trabalho ou a realização de seus valores por meio dessa atividade, sendo uma emoção positiva de bem-estar.

Herzberg (1966) desenvolveu uma teoria motivacional, a qual chamou de "Teoria dos dois Fatores" onde abordou a satisfação e a insatisfação no trabalho como fenômenos distintos. O autor colocou que a insatisfação está relacionada aos fatores que determinam o trabalho (fatores extrínsecos), como ambiente, condições de trabalho, políticas de remuneração, relacionamento, entre outros. Por outro lado, vinculou a satisfação no trabalho como fatores intrínsecos, e que esses podem ser encontrados no conteúdo do trabalho e se refletem diretamente no que a pessoa faz diariamente no trabalho. Incluem fatores como o senso de realização, reconhecimento, responsabilidade, chances de promoção e oportunidades de crescimento pessoal, entre outros (SCHERMERHORN; HUNT; OSBORN, 1999).

Sob um prisma mais psicológico, Locke (1976) inferiu que a satisfação advém de um estado emocional positivo ou de prazer, resultante da avaliação do trabalho ou das experiências proporcionadas pelo trabalho. Na acepção de Brief (1988, p.86), a satisfação é um "estado emocional expresso a partir da avaliação afetiva e/ou cognitiva de uma experiência de trabalho, com algum grau de favorabilidade ou de desfavorabilidade". E nesse sentido, Seal e Knight (1988) complementaram que a satisfação no trabalho significa as respostas emocionais ou avaliação global dos funcionários para o trabalho em si. Estes autores apontam falhas em algumas teorias desse tema, como a de Herzberg (1966) quem considera satisfação e insatisfação fenômenos distintos, não indicando as diferenças individuais de percepção da satisfação no trabalho, justificando essa falha com a afirmativa de que os valores são pessoais e que podem ser determinantes no trabalho.

George e Jones (1999) vislumbraram a satisfação no trabalho como sendo um conjunto de sentimentos e crenças dos indivíduos sobre o seu atual trabalho. Os autores ainda enfatizaram que os níveis de satisfação das pessoas sobre o seu trabalho podem ir da satisfação extrema à insatisfação extrema, sendo que esses níveis interferem nas atitudes dessas sobre o seu trabalho como um todo, como o tipo de trabalho que fazem, com os seus colegas, supervisores ou subordinados, entre outros. Em seguida, tem-se Robbins (2002), o qual definiu satisfação no trabalho como a atitude geral que as pessoas têm em relação ao seu trabalho, ou seja, as pessoas pos- 
suem uma reação ativa às situações de trabalho que não o satisfazem, deliberando atitudes em busca de mudanças.

Tratando da importância que a satisfação tem na vida das pessoas, Rozhowski e Hulin (1992) sugeriram que as organizações devem levar em consideração o nível de satisfação no trabalho, pois esse apresenta-se como um fator importante no ambiente organizacional. Da mesma forma, entretanto de maneira mais genérica, Spector (1997), enfatiza que, de uma maneira geral, toda a sociedade é beneficiada quando as pessoas se sentem satisfeitas com o seu trabalho.

Diante do breve resgate de algumas definições sobre o tema da satisfação no trabalho, ressalta-se o apanhado de Marqueze e Moreno (2005), os quais enfatizaram que alguns autores consideram a satisfação no trabalho como um estado emocional, um sentimento, conforme apresentado anteriormente (LOCKE, 1969, 1976; HENNE; LOCKE, 1985; HARRIS, 1989; BEGLEY; CZAJKA, 1993; FRASER, 1996; WRIGHT; CROPANZANO, 2000; ELOVAINIO et. al., 2000; O'DRISCOLL; BEEHR, 2000). Outros consideram satisfação como uma atitude (REGO, 2001; ROBBINS, 2002). Essas diferenças podem resultar em erros metodológicos de pesquisa e devem ser observadas e consideradas quando da escolha do instrumento de medida para ir ao encontro dos objetivos propostos.

Buscou-se em Bergamini e Beraldo (1988), o fechamento dessa enxuta sobre a satisfação com o trabalho, onde os autores elucidam que as definições sobre satisfação no trabalho, independentemente da concepção abordada, só possuem validade se forem consideradas as diferenças individuais resultantes das variáveis inatas. Seguindo esse raciocínio, faz-se uma conexão aos valores humanos por serem princípios enraizados dificilmente modificáveis os quais norteiam a vida dos indivíduos (SCHWARTZ, 2005); e, portanto, acredita-se que esta seja uma abordagem apropriada para se estudar a satisfação no trabalho.

Além disso, a ideia defendida por alguns autores de satisfação como atitude pode ser conectada à de Schwartz (2005) de que valores podem fornecer poder preditivo e explicativo na análise das atitudes. Nesse sentido, na seção seguinte é apresentada a teoria de valores humanos a qual fundamenta este estudo.

\subsection{Valores Pessoais}

Valores humanos são guias que orientam as atitudes e os comportamentos das pessoas (SCHWARTZ; BILSKY, 1987) e são um tema de estudo recorrente em pesquisas na Psicologia Social (GRUNERT; JUHL, 1995), entretanto, passou a ser um tema de estudo também na área de Administração com a popularização da teoria de valores pessoais de Shalom Schwartz (CASTRO, 2006; SCHWARTZ, 1994; SCHWARTZ, 1992), perspectiva adotada para o presente ensaio. Teóricos que antecederam Schwartz propuseram conceitos diversos sobre o tema, compartilhando da ideia de universalidade dos valores (KLUCKHOHN, 1951; ROKEACH, 1973). A teoria de Schwartz também adota pressupostos de universalidade ao propor que "valores são concepções do desejável que guiam maneiras como atores sociais selecionam ações e avaliam pessoas e eventos, explicando suas ações e avaliações" (SCHWARTZ, 1994, p. 2).

A Teoria de Valores Pessoais de Schwartz $(1994,1992)$ especifica um conjunto de valores de ordem superior e de ordem inferior. Os 10 valores de ordem inferior formam os 4 valores de ordem superior, os quais estão organizados em uma estrutura circular de duas dimensões bipolares. Em uma dimensão estão os valores "Abertura à mudança" versus "Conservação", os quais, segunda a teoria, possuem uma relação de oposição. Na outra dimensão estão os valores "Autopromoção" versus "Autotranscendência" (Figura 1). 


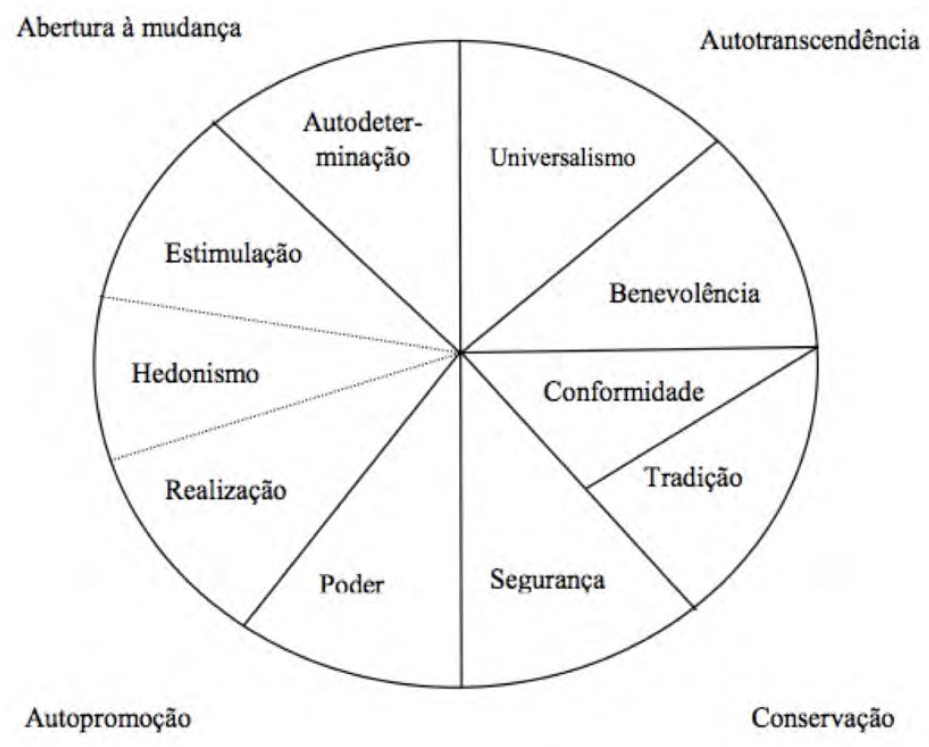

Figura 1. Estrutura de Valores Pessoais de Schwartz

Fonte: Schwartz (1992, p. 45)

Esta estrutura teorizada tem sido suportada nos estudos realizados em diversos países, desde 2002, pelo Inquérito Social Europeu (European Social Survey - ESS); assim como em outros estudos os quais utilizaram o escalonamento multidimensional (BILSKY; JANIK; SCHWARTZ, 2010; SCHWARTZ 2006).

Para melhor entendimento, no Quadro 1 o conjunto dos 10 valores de ordem inferior estão distribuídos entre os 4 valores de ordem superior com a descrição de suas metas motivacionais as quais orientam os indivíduos ao longe de suas vidas. Ressalta-se que, conforme se pode observar a partir da linha tracejada na estrutura dos valores (Figura 1), o valor de ordem inferior "Hedonismo" é compartilhado com a Autropromoção e a Abertura à Mudança e, portanto, nesta pesquisa, foi considerado como variável que constrói cada um desses fatores.

Quadro 1. Valores de ordem superior e inferior e metas motivacionais

\begin{tabular}{|c|c|}
\hline CONJUNTO DE VALORES & METAS MOTIVACIONAIS \\
\hline Abertura a Mudança & Autodireção e Estimulação \\
\hline 1 Autodeterminação & Independência de pensamento e ação; criar e explorar. \\
\hline 2 Estimulação & $\begin{array}{c}\text { Manter-se em excelente nível de ativação; excitação, novidade e de- } \\
\text { safio na vida. }\end{array}$ \\
\hline 3 Hedonismo & $\begin{array}{c}\text { Prazer, procura satisfazer seus desejos; curte os prazeres da vida, quer } \\
\text { gozar a vida. }\end{array}$ \\
\hline Autopromoção & Hedonismo, Realização e Poder \\
\hline
\end{tabular}




\begin{tabular}{|c|c|}
\hline 3 Hedonismo & $\begin{array}{l}\text { Prazer, procura satisfazer seus desejos; curte os prazeres da vida, quer } \\
\text { gozar a vida. }\end{array}$ \\
\hline 4 Realização & Realizar padrões internos de excelência. \\
\hline 5 Poder & Status social e prestígio. \\
\hline Conservação & Conformidade, Tradição e Segurança \\
\hline 6 Conformidade & Restringir ações, inclinações e impulsos socialmente indesejáveis. \\
\hline 7 Tradição & $\begin{array}{l}\text { Respeito, compromisso e aceitação dos costumes e ideias que uma cul- } \\
\text { tura impõe ao indivíduo. }\end{array}$ \\
\hline 8 Segurança & Estabilidade da sociedade, das relações e da própria pessoa. \\
\hline Autotranscendência & Universalismo e Benevolência \\
\hline 9 Universalismo & $\begin{array}{l}\text { Entender, apreciar, tolerar e proteger tendo em vista o bem-estar da } \\
\text { humanidade e da natureza. }\end{array}$ \\
\hline 10 Benevolência & Preservação e bem-estar das pessoas mais próximas. \\
\hline
\end{tabular}

Fonte: Schwartz (1992)

É de comum acordo entre diversos teóricos (SCHWARTZ, 2006; HALMAN; DE MOOR, 1994; SCHWARTZ, 1992; ROKEACH, 1973); a visão de valores como crenças, profundamente enraizadas, as quais explicam comportamentos, escolhas, atitudes, opiniões e ações, e também orientam os indivíduos no julgamento que eles fazem de situações (SCHWARTZ, 1994). Como determinantes do comportamento humano, os valores pessoais são elementos importantes também na relação de um indivíduo com o seu trabalho (TAMAYO, 2001). O elo que se faz entre os "valores pessoais" e sua influência na satisfação no trabalho é reforçado a partir da visão de Brief (1988) de que a satisfação no trabalho envolve sentimentos e crenças. Nesse sentido, os valores são neste estudo vistos como fatores explicativos da satisfação humana no trabalho, e, portanto, buscou-se investigar essa influência.

\subsection{Relação entre Valores Pessoais e Satisfação no Trabalho}

Schwartz (2005) menciona o valor como um "princípio guia" o qual possui um caráter antecedente às escolhas, atitudes, avaliações e ações humanas. Em sua teoria, ele sugere que os valores humanos sejam universais por serem baseados em requisitos básicos para a existência humana e são concepções profundamente enraizadas, ou seja, dificilmente modificadas.

Cabe ressaltar que o sistema de valores pessoais é "nada mais do que uma disposição hierárquica de valores, uma classificação ordenada de valores ao longo de um contínuo de importância" (ROKEACH, 1969, p. 551). A organização hierárquica de valores pressupõe que o indivíduo não se relaciona com o mundo físico e social como observador que assiste a um espetáculo, mas como ator que participa, que toma partido, que se envolve nele. Os valores implicam necessariamente uma preferência e distinção entre o que é importante para o indivíduo e o que é secundário, entre o que tem valor e o que não tem. Assim, as prioridades axiológicas da pessoa definem a sua visão do mundo, do trabalho, do relacionamento com os colegas e determinam o leque de interesses na vida e no trabalho (TAMAYO, 2001). 
Conforme já abordado anteriormente, na Teoria de Valores Pessoas de Schwartz (1992, 1994), os valores pessoais se resumem em quatro valores com relação de oposição entre os valores de cada dimensão. Assim como valor de autotranscendência se opõem ao valor de autopromoção, o valor de conservação se opõe ao valor de abertura à mudança. Essas afirmações são corroboradas com as de Tamayo (2001) quem infere a existência de uma relação estrutural básica entre os valores e entre os tipos motivacionais por eles constituídos, e que foram sintetizadas mediante duas dimensões bipolares, já verificadas empiricamente tanto no Brasil (TAMAYO, 1993; TAMAYO; SCHWARTZ, 1993; TAMAYO, 1994) como em diversos países na Europa (ESS, 2012; SCHWARTZ, 1994, 1992).

A autotranscendência e a conservação apresentam, respectivamente, características de universalismo e tradição. Pessoas com estas características são menos ambiciosas e mais tolerantes; seus interesses transcendem aos seus próprios interesses; elas acreditam que as pessoas deveriam estar satisfeitas como o que elas possuem (SCHWARTZ, 1994, 1992). Tanto autotranscendentes quanto conservadores podem apresentar maior grau de satisfação com sua vida como um todo, o que nos sugere incluir a satisfação com seu trabalho também. Ressalta-se que Tamayo (2001) estudou a relação dos valores pessoais com a satisfação no trabalho e constatou que os valores de autotranscendência influenciam a satisfação com os colegas de trabalho, e a prioridade dada aos valores de conservação afetam a satisfação com a chefia e a satisfação com os colegas (TAMAYO, 2001).

Diante do exposto formou-se a primeira hipótese de investigação deste estudo:

Hipótese 1: Há uma relação positiva entre o valor de autotranscendência e a satisfação com o trabalho.

Na acepção de Tamayo (2001), a dimensão autopromoção versus autotranscendência, apresenta, nos respectivos extremos que se encontram, os valores relativos aos tipos motivacionais "poder", "realização" e "hedonismo"; e os valores de "universalismo" e "benevolência". Este eixo ordena os valores com base na motivação da pessoa para promover os seus próprios interesses mesmo às custas dos outros, por oposição a transcender as suas preocupações egoístas e promover o bem-estar dos outros e da natureza. Essas afirmações vão de encontro com Schwartz $(1994,1992)$, quem coloca, em sua teoria, que os valores de polos opostos possuem uma relação de oposição e consequente associação negativa, o que nos permite supor que se um valor possui uma relação positiva com determinado fator, o valor diametralmente oposto a ele apresentaria uma relação negativa com este mesmo fator. Portanto, com base neste argumento se constrói a hipótese seguinte.

Hipótese 2: Há uma relação negativa entre o valor de autopromoção e a satisfação com o trabalho

Por outro lado, na dimensão abertura à mudança versus conservação, os valores são ordenados, respectivamente, com base na motivação da pessoa a seguir os seus próprios interesses intelectuais e afetivos através de caminhos incertos e ambíguos, com oposição à tendência a preservar o status quo e a segurança que ele gera no relacionamento com os outros e com as instituições. Teoricamente, situam-se, no polo primeiro polo deste eixo, os valores relativos aos tipos motivacionais "estimulação", "autodeterminação" e "hedonismo"; e no outro, os valores referentes à "segurança", "conformidade" e "tradição" (TAMAYO, 2001; Schwartz, 2005). Nesse sentido, as hipóteses seguintes referem-se ao valor de conservação, o qual, entre outras características, tem como base a concepção de que as pessoas deveriam estar satisfeitas como o que elas possuem, assim como por conseguinte, a hipótese da abertura à mudança, a qual foi formulada com base no conceito de oposição conceitual e associação negativa entre os valores de polos 
opostos (SCHWARTZ, 1994, 1992).

Hipótese 3: Há uma relação positiva entre o valor de conservação e a satisfação com o trabalho

Hipótese 4: Há uma relação negativa entre o valor de abertura à mudança e a satisfação com o trabalho

\section{METODOLOGIA}

A presente pesquisa é de cunho quantitativo e utilizou dados secundários do ano de 2012 retirados da base de dados do Inquérito Social Europeu (ESS, 2012). O ESS aborda diversas questões sociais em um conjunto de 20 países europeus, dentre os quais está incluído Portugal, o qual foi eleito para ser trabalhado neste artigo. Em Portugal, o ESS é conduzido pelo Instituto de Ciências Sociais da Universidade de Lisboa (ICS) e pelo Instituto Superior de Ciências (ISCTE-IUL). A base de dados selecionada possui uma amostra válida de 820 participantes portugueses, de um total de 2.151, com idade mínima de 15 anos e máxima de 97 anos.

O conjunto de valores de Schwartz $(1994,1992)$ é composto por 10 constructos (vistos no Quadro 1 da seção 2), sendo que cada um deles é caracterizado por 21 afirmativas específicas do Portrait Questionare Values (PQV-21) e estas são as variáveis encontradas na base de dados utilizada. Como os valores pessoais não são diretamente observáveis, foram criadas as variáveis para medi-los, de acordo com a própria teoria de Schwartz (1992, 1994). Portanto, essas 21 questões são agrupadas em 10 variáveis observáveis formadas da seguinte forma: 1) Autodeterminação inclui as afirmativas 1 e 11; 2) Estimulação, as afirmativas 6 e 15; 3) Hedonismo, as afirmativas 10 e 21; 4) Realização, as afirmativas 4 e 13; 5) Poder, as afirmativas 2 e 17; 6) Conformidade, as afirmativas 7 e 16; 7) Tradição, as afirmativas 9 e 20; 8) Segurança, as afirmativas 5 e 14; 9) Universalismo, as afirmativas 3,8 e 19; 10) Benevolência, as afirmativas 12 e 18 (SCHWARTZ, 1992, 1994).

Neste estudo, as 21 variáveis foram agrupadas diretamente nos 4 valores de ordem superior (abertura à mudança, conservação, autopromoção e autotranscendência), pois o objetivo foi trabalhar com estes valores e não os de ordem inferior. A construção dessas variáveis se deu com base na teoria (Schwartz, 1992, 1994), a partir do software SPSS, por meio do cálculo das médias das respostas de cada uma das 21 afirmativas encontradas na base de dados. Na Figura 2 é demonstrado um esquema com a composição das variáveis de forma facilitar o entendimento. Portanto, a Abertura à mudança foi formada a partir da média das variáveis (1) autodeterminação, (2) estimulação e (3) hedonismo. A Conservação, de (6) conformidade, (7) tradição e (8) segurança. A Autopromoção, de (3) hedonismo, (4) realização e (5) poder. Por fim, a Autotranscendência, a partir da média de (9) Universalismo e (10) benevolência.

Dessa maneira foi possível medir os quatro valores de ordem superior como variáveis independentes no modelo de Regressão Linear Múltipla mostrado posteriormente. 


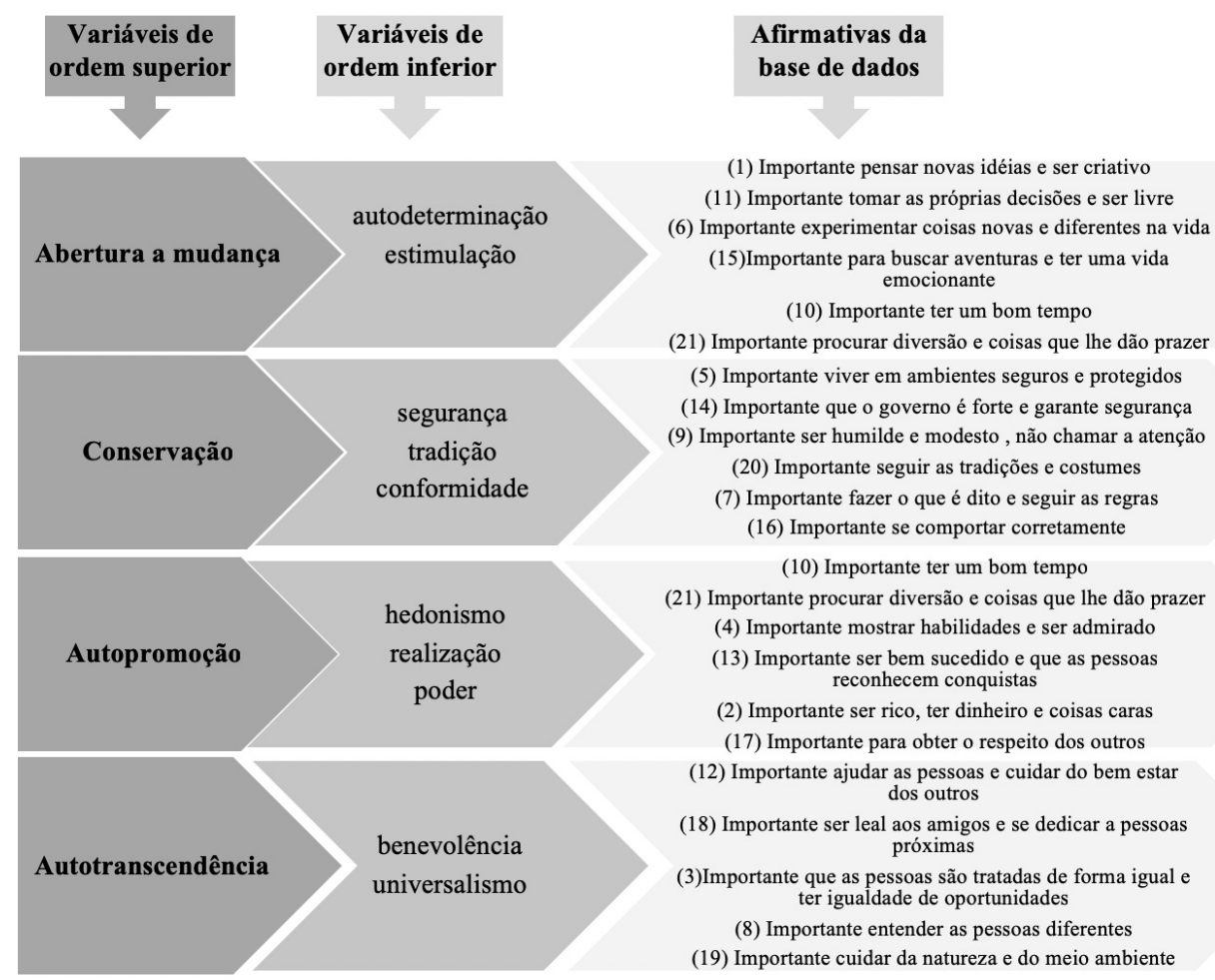

Figura 2. Esquema de composição das variáveis

Fonte: Adaptado de Schwartz (1992)

Além das quatro variáveis, utilizou-se também para este ensaio a variável ordinal denominada "Quão satisfeito estou com o trabalho", a qual compôs o modelo, conforme mostrado a seguir.

$$
Y=\beta_{1}+\beta_{2} S T+\beta_{3} S E+\beta_{4} C O+\beta_{5} O C+E
$$

Nesta regressão, a variável dependente $(\mathrm{Y})$ diz respeito à Satisfação com o Trabalho (ST); e as variáveis independentes referem-se, respectivamente, aos 4 valores pessoais: Autotranscedência (AT), Autopromoção (AP), Conservação (CO), e Abertura à Mudança (AM). Para melhor entendimento da estrutura do modelo testado neste estudo, segue o desenho do modelo com as devidas variáveis e hipóteses de pesquisa.

\subsection{Desenho do Modelo}

Diante das hipóteses formuladas anteriormente, é apresentada nesta seção a proposta para o modelo deste estudo. Conforme visto na Figura 2, o modelo apresenta os quatro valores pessoais de ordem superior como variáveis independentes e, portanto, explicativas; e à direita, a variável dependente do modelo, com as respectivas hipóteses de pesquisa. 


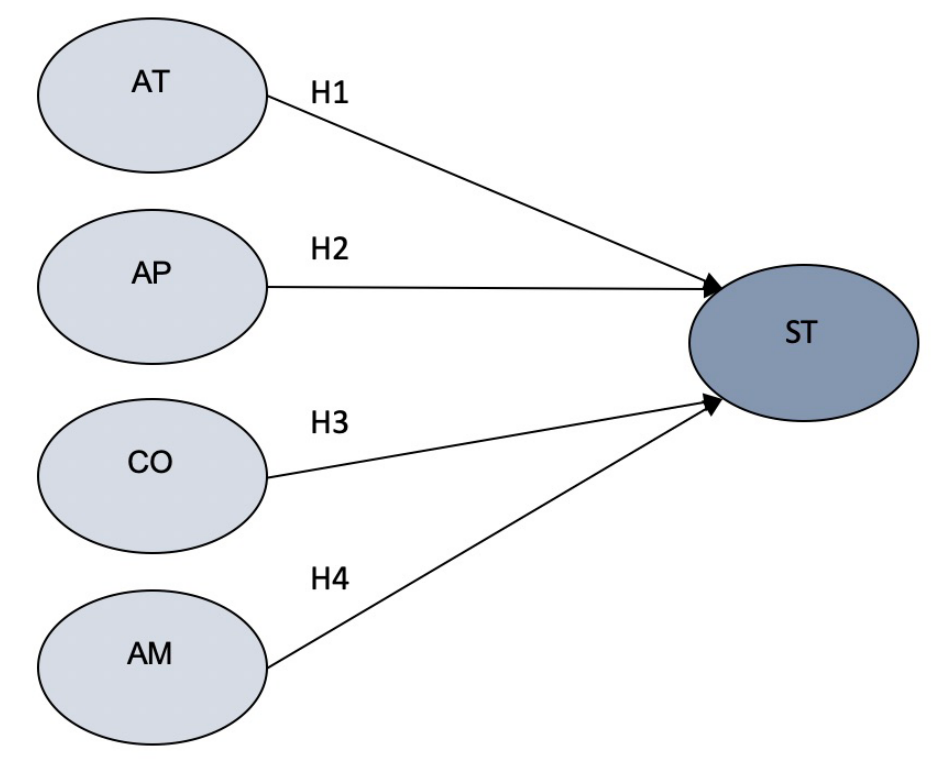

Figura 3: Modelo proposto

Nota: AT - Autotranscendência; AP - Autopromoção; CO - Conservação; AM - Abertura a Mudança, ST - Satisfação no Trabalho

\subsection{Análise estatística}

Como o estudo em questão teve o interesse de verificar a satisfação do trabalho em função de 4 variáveis explicativas formadas pelos valores pessoais, optou-se por utilizar como técnica de análise a Regressão Linear Múltipla a partir do software SPSS (Statistic Package for Social Sciences), versão 22 para Windows, por ser uma técnica utilizada quando se pretende verificar a relação linear entre duas ou mais variáveis independentes (explicativas) e uma dependente (explicada), (MARÔCO, 2014).

\section{RESULTADOS E DISCUSSÃO}

Os resultados deste estudo foram obtidos a partir da análise de regressão linear múltipla, entretanto, antes de seguir com a análise foram verificados e confirmados os pressupostos básicos (linearidade, homocedasticidade, independência dos erros e sua distribuição normal), (MARÔCO, 2014; TABACHNICK e FIDELL, 2001).

Observou-se por meio dos dados demográficos uma amostra em sua maioria feminina $(60,4 \%)$ e com uma média de idade de 52,29 anos. Quanto à escolaridade, notou-se que $69,6 \%$ dos participantes possui apenas o ensino básico, enquanto $5,1 \%$ possuem o ensino superior e 7,9\% pós-graduação. A estatística descritiva das variáveis é apresentada na Tabela 1.

Tabela 1: Estatística descritiva dos dados 


\section{Estatística Descritiva}

\begin{tabular}{lcc}
\hline Variáveis & Média & Desvio Padrão \\
Satisfação com o trabalho & 7,08 & 1,847 \\
ST & 2,3407 &, 65897 \\
SE & 2,9961 &, 70409 \\
CO & 2,7404 &, 74473 \\
OC & 2,9059 &, 69490 \\
N & & 820 \\
\hline
\end{tabular}

Note: $\mathrm{N}$ - Amostra válida.

O modelo deste estudo considerou exclusivamente os valores pessoais de Schwartz como variáveis independentes, e a "satisfação com o trabalho" como variável dependente. A análise dos resultados mostrou que o coeficiente de correlação $\mathrm{R}$ do modelo revelou uma correlação positiva, fraca $(0,179)$ e significativa ao nível de $5 \%$; e o coeficiente de determinação $\left(R^{2}\right)$ revelou que $3,2 \%$ da variância total da satisfação no trabalho é explicada pelo modelo; portanto os $97 \%$ restantes dessa variação são explicadas por outros fatores, notando-se, assim, a necessidade de incluir mais variáveis explicativas ao modelo.

Com respeito às hipóteses de investigação (Tabela 2 ), notou-se que a Hipótese 1 foi rejeitada ( $\beta=-0,079 ; p>0,05)$, e, portanto, não há uma relação positiva entre autotranscendência e a satisfação com o trabalho. Esse resultado não corrobora com o de Tamayo (2001) quem identificou uma relação positiva entre esse valor com a satisfação no trabalho com a chefia e com os colegas.

A segunda hipótese da pesquisa foi formulada com base no conceito de relação de oposição entre valores da mesma dimensão e, portanto, esperava-se uma relação negativa com a satisfação no trabalho. Entretanto, isso não foi constatado e rejeitou-se também a Hipótese 2 de que haveria uma relação negativa entre autopromoção e a variável a ser explicada $(\beta=-0,012$; $p>0,05)$.

A Hipótese 3 deste estudo também foi rejeitada $(\beta=-0,003 ; p>0,05)$ revelando não haver relação positiva, tampouco negativa significante, entre conservação e a satisfação com o trabalho. Embora a variável dependente do estudo de Tamayo (2001) não tenha sido a satisfação com o trabalho de forma geral, como o foi neste estudo, seu estudo sugeriu um resultado diferente do que foi visto aqui, no qual o valor de conservação apresentou uma relação significativa com a satisfação com a chefia e com os colegas de trabalho. Porto e Tamayo $(2007$, p.6) também incluíram em seu estudo o valor de conservação, entretanto, sugerindo que este pudesse ter associação negativa com a Realização Profissional, a qual não foi sustentada; mas que não reforça o resultado do presente estudo por ser uma variável que está mais relacionada "à busca de prazer e realização pessoal e profissional, bem como de independência de pensamento e ação no trabalho por meio da autonomia intelectual e da criatividade".

Por fim, não rejeita-se a Hipótese 4, cuja análise revelou uma relação negativa e estatis- 
ticamente significante ao nível de $5 \%(-0,116 ; p<0,05)$. Todavia, esta hipótese foi construída com base na relação de oposição dos valores em uma mesma dimensão (Schwartz, 1994). O estudo de (PORTO; TAMAYO, 2007), apresentou um resultado positivo do valor abertura à mudança ao fato de sentir-se realizado no trabalho. Embora, sentir-se realizado e sentir-se satisfeito no trabalho sejam variáveis diferentes, pode-se sugerir que ser aberto à mudança pode proporcionar um sentimento positivo com o trabalho, refletindo esses dois fatores.

Tabela 2: Coeficientes do modelo de regressão linear múltipla

\begin{tabular}{|c|c|c|c|c|c|c|c|c|}
\hline \multicolumn{9}{|c|}{ Coeficientes } \\
\hline & \multirow{2}{*}{ Modelo } & \multicolumn{2}{|c|}{$\begin{array}{l}\text { Coeficientes não } \\
\text { padronizados }\end{array}$} & \multicolumn{3}{|c|}{ Coeficientes padronizados } & \multicolumn{2}{|c|}{$\begin{array}{l}95,0 \% \text { Intervalo de } \\
\text { confiança para B }\end{array}$} \\
\hline & & B & $\begin{array}{l}\text { Std. } \\
\text { Error }\end{array}$ & Beta & $\mathrm{t}$ & Sig. & $\begin{array}{l}\text { Limite } \\
\text { Inferior }\end{array}$ & $\begin{array}{l}\text { Limite } \\
\text { Superior }\end{array}$ \\
\hline \multirow{5}{*}{1} & Constant) & 8,616 &, 320 & & 26,885 & ,000 & 7,987 & 9,245 \\
\hline & ST &,- 222 & ,125 &,- 079 & $-1,774$ & ,076 &,- 469 &, 024 \\
\hline & SE &,- 032 & 155 &,- 012 &,- 206 &, 837 &,- 337 &, 273 \\
\hline & $\mathrm{CO}$ &,- 007 & , 108 &,- 003 &,- 068 & ,946 &,- 219 & ,204 \\
\hline & $\mathrm{OC}$ &,- 308 & ,155 &,- 116 & $-1,989$ & ,047 &,- 613 &,- 004 \\
\hline
\end{tabular}

a. Variável dependente: Satisfação com o trabalho

Fonte: Software SPSS $22.0^{\circledR}$.

De forma geral, os resultados mostraram que os valores pessoais não possuem relação com a satisfação no trabalho, com exceção do valor de abertura à mudança o qual revelou uma relação negativa significante, embora fraca e com uma suposição baseada exclusivamente no conceito de oposição entre valores da mesma dimensão sugerido pela teoria utilizada neste estudo. Esses resultados se assemelham com os de Soraggi e Paschoal (2011) quem buscaram entender se os valores pessoais de Schwartz $(1992,1994)$ seriam preditores do bem-estar no trabalho, entretanto, seus resultados sugeriram não haver relação positiva entre essas variáveis.

Uma vez que os valores pessoais não são fatores com considerável poder explicativo para a satisfação com o trabalho, percebe-se que outros fatores individuais (intrínsecos) e contextuais (extrínsecos) poderiam ser acatados para verificar a satisfação com o trabalho. Com base neste argumento é possível encontrar na literatura pertinente, estudos desenvolvidos os quais buscaram conceituar, explicar e medir a satisfação no trabalho.

Toma-se como exemplo o estudo de Herzberg (1966), quem desenvolveu a Teoria de dois Fatores - uma teoria motivacional, na qual abordou a satisfação e a insatisfação no trabalho, sendo esses, fenômenos distintos. O autor sugeriu que a insatisfação está relacionada aos fatores que determinam o trabalho (fatores extrínsecos), como ambiente, condições de trabalho, políticas de remuneração, relacionamento, entre outros. Por outro lado abordou a satisfação no trabalho advém de fatores intrínsecos, e inferiu que esses podem ser encontrados no conteúdo do trabalho e se refletem diretamente no que a pessoa faz diariamente no trabalho, como, por exemplo, o senso de realização, reconhecimento, responsabilidade, chances de promoção e oportunidades de crescimento pessoal, entre outros (SCHERMERHORN, HUNT E OSBORN, 1999). 
Em seu estudo sobre a percepção dos subordinados sobre os estilos de liderança e seu comportamento, Suar, Tewari e Chaturbedi (2006) também levaram em consideração para explicar a satisfação no trabalho os aspectos intrínsecos como níveis de responsabilidade, autonomia, progressão funcional, entre outros; assim como extrínsecos, tais como o trabalho desafiador, o salário, as horas de trabalho, o tipo de cargo, dentre outros relacionados à sua atuação no ambiente organizacional.

Outros aspectos contextuais (contexto organizacional) também foram considerados na literatura para explicar a satisfação com o trabalho, como visto no estudo de Seltzer, Alone e Howard (1996), no qual os autores propuseram que a satisfação no trabalho refere-se às atitudes de um indivíduo no que tange à sua percepção sobre fatores contextuais relacionados à sua função, tais como o apoio constante dos colegas e supervisores à aplicação de novas habilidades no trabalho, o grau de diversidade e complexidade da tarefa, a percepção de trabalho desafiador, autonomia e acompanhamento contínuo do desempenho.

Sob um aspecto mais amplo, notam-se estudos os quais demonstraram a interferência de fatores externos que influenciam a satisfação das pessoas no trabalho. Esses aspectos são investigados principalmente quando são desenvolvidas pesquisas de Clima Organizacional, nas quais se buscam descobrir o nível de satisfação humana no ambiente de trabalho. Entretanto, algumas investigações levam em consideração os fatores externos à organização e que influenciam o ser humano nas suas percepções com relação ou seu trabalho e ao significado que este tem na sua vida, podendo, a partir daí, inferir uma maior ou menor satisfação. Nesse aspecto, Bispo (2006) fez um apanhado em alguns modelos de pesquisa de clima e verificou que além de fatores externos ao indivíduo, porém internos no ambiente organizacional, os modelos levam em consideração a vida profissional, o ambiente de trabalho, assistência aos funcionários, a burocracia, a cultura organizacional, a estrutura organizacional, o nível sociocultural os incentivos, a remuneração, e o transporte. Além disso, autor identificou e incluiu em seu modelo fatores ambientais externos, ou seja, aqueles que são externos ao ambiente organizacional e também externos ao indivíduo, e que acabam por interferir na sua satisfação no trabalho, tais como a convivência familiar, lazer, investimentos e despesas familiares, política e economia, saúde, segurança pública, e situação financeira.

Esses aspectos corroboraram com a ideia de Fraser (1984) de que a satisfação no trabalho é um fenômeno complexo e de difícil definição. Uma parte dessa dificuldade decorre do fato de que a satisfação no trabalho é um estado subjetivo e que, portanto, pode variar de pessoa a pessoa, de circunstância para circunstância, e pode estar sujeita a influências de forças internas e externas ao ambiente de trabalho imediato. Assim como também de Kanaane (1995) - quem apresenta um paradigma mais globalizado e holístico sobre o trabalho - e enfatiza que esse (o trabalho) ultrapassa os limites sócio-políticos, sendo caracterizado por uma relação mais humanizada entre seus componentes.

Essa breve retomada de estudos nos leva a considerar que em estudos sobre satisfação no trabalho é relevante considerar a complexidade dos fatores que interferem a percepção do ser humano, e que, portanto, a inclusão de outras variáveis explicativas se faz necessária. Esse argumento advém do fundamento de Hoppock (1935) - autor seminal do tema em questão - o qual considerou a satisfação no trabalho como uma manifestação sentimental do ser humano no ambiente de trabalho e que pode estar ligada ao campo tanto físico como psicológico 


\section{CONSIDERAÇÕES FINAIS}

O objetivo deste estudo foi analisar a relação dos valores pessoais e a satisfação com o trabalho entre a população portuguesa e para isso foram construídas quatro hipóteses de investigação, sugerindo que entre os quatro valores pessoais de Schwartz $(1992,1994)$, a autotranscedência e a conservação seriam valores que poderiam ter uma relação positiva com a satisfação com o trabalho, assim como os valores de oposição a estes (autopromoção e abertura à mudança), teriam relação negativa. Contudo, a partir da análise realizada, notou-se a irrelevância dos valores pessoais utilizados neste estudo no sentido de explicarem a satisfação no trabalho nesta amostra.

Como pode ser observado na discussão dos resultados, vários fatores vinculados ao indivíduo assim como o contexto poderiam ter sido experimentadas no modelo com o intuito forçar uma relação mais significativa de determinados fatores (principalmente pessoais) com a satisfação no trabalho. No entanto, este não foi o objetivo deste estudo o qual foi sustentado no entendimento sobre os valores pessoais da Teoria de valores de Schwartz $(1992,1994)$. Contudo, isso é colocado como limitação deste estudo, ao destacar, portanto, a utilização de um único modelo contendo valores pessoais como variáveis explicativas da satisfação no trabalho.

Sugere-se, assim, para futuras pesquisas, a inclusão de outras variáveis explicativas no modelo estimado, sejam variáveis individuais como personalidade, altruísmo, senso de realização, entre outros; e/ou variáveis contextuais como perfil do líder, setor da empresa, cargo e tempo de trabalho, a fim de verificar o poder explicativo destas variáveis sobre a satisfação no trabalho. Além disso, sugere-se também incluir no modelo as variáveis demográficas da amostra, pois os valores podem variar de acordo com a idade, gênero, e grau de escolaridade (SOARES; AFONSO, 2014; DAEHLEN, 2007).

Com este estudo espera-se contribuir com acadêmicos que estudam a satisfação no trabalho a partir de variáveis individuais, ao sugerir que valores pessoais não tiveram poder explicativo significante sobre essa variável, na amostra estudada, e que, portanto, sugere-se trabalhar com modelos mais complexos que incluam características individuais dos participantes. Como indicação para a prática, aconselha-se aos gestores que considerem outras variáveis individuais como determinantes para a satisfação no trabalho.

\section{REFERÊNCIAS}

BEGLEY, Thomas M.; CZAJKA, Joseph M. Panel analysis of the moderating effects of commitment on job satisfaction, intent to quit, and health following organizational change. Journal of Applied psychology, v. 78, n. 4, p. 552, 1993.

BERALDO, Deobel Garcia Ramos; BERGAMINI, Cecília Whitaker. Avaliação de desempenho humano na empresa. 1988.

BISPO, Carlos Alberto Ferreira. Um novo modelo de pesquisa de clima organizacional. Revista Produção, v. 16, n. 2, p. 258-273, 2006. BISPO, Carlos Alberto Ferreira. Um novo modelo de 
pesquisa de clima organizacional. Revista Produção, v. 16, n. 2, p. 258-273, 2006.

ARTHUR P. BRIEF. Attitudes in and around organizations. Sage, 1998.

CASTRO SOLANO, Alejandro; NADER, Martín. La evaluación de los valores humanos con el Portrait Values Questionnaire de Schwartz.Interdisciplinaria, v. 23, n. 2, p. 155-174, 2006.

CHRYSSOHOIDIS, George; KRYSTALLIS, Athanasios. Consumers' willingness to pay for organic food: Factors that affect it and variation per organic product type. British Food Journal, v. 107, n. 5, p. 320-343, 2005.

DAEHLEN, Marianne. Job values, gender and profession: A comparative study of the transition from school to work. Journal of Education and Work, v. 20, n. 2, p. 107-121, 2007.

EALIAS, Abi; GEORGE, Jijo. Emotional intelligence and job satisfaction: a correlational study. Research Journal of Commerce and Behavioral Science, v. 1, n. 4, 2012.

ELOVAINIO, Marko et al. Organizational and individual factors affecting mental health and job satisfaction: a multilevel analysis of job control and personality. Journal of occupational health psychology, v. 5, n. 2, p. 269, 2000.

ROUND, E. S. S. 6: European Social Survey Round 6 Data (2012). Data file edition, v. 1.

FRASER, TM. Human stress, work and job satisfaction: A critical approach. In: Occupational Safety and Health Series, v. 50, p. 13-16, 1984.

FRASER, T. M. Work, fatigue, and ergonomics. In: INTRODUCTION to industrial ergonomics: a textbook for students and managers (online). Toronto: Wall and Emerson, 1996. Available from: http://www. wallbooks.com/source/fraser.htm. Access in: 2015 jan 10.

GEORGE, Jennifer M.; JONES, Gareth R. Understanding and managing organizational behavior. (2nd ed.), Reading, MA: Addison-Wesley, 1999.

GRUNERT, Suzanne C.; JUHL, Hans Jørn. Values, environmental attitudes, and buying of organic foods. Journal of economic psychology, v. 16, n. 1, p. 39-62, 1995.

HACKMAN, J.R.; OLDMAN, G. R. Work Redesign. Reading, MA: Addison Wesley, (1980).

ESTER, Peter; HALMAN, Loek; DE MOOR, Ruud. Value shift in Western societies. The individualizing society: Value change in Europe and North America, p. 1-20, 1993.

HARRIS, Ruth Beall. Reviewing nursing stress according to a proposed coping-adaption framework. Advances in Nursing Science, v. 11, n. 2, p. 12-28, 1989.

HASNAH HASSAN, Siti. Managing conflicting values in functional food consumption: the Malaysian experience. British Food Journal, v. 113, n. 8, p. 1045-1059, 2011.

HENNE, Douglas; LOCKE, Edwin A. Job dissatisfaction: what are the consequences?. International Journal of Psychology, v. 20, n. 2, p. 221-240, 1985.

HERZBERG, Frederick I. Work and the nature of man. 1966.

HOPPOCK, R. A satisfação no trabalho. New York: Harper \& Irmão, 1935. 
KANAANE, Roberto. Comportamento humano nas organizaçöes: o homem rumo ao século XXI. In: Comportamento humano nas organizaçöes: o homem rumo ao século XXI. Atlas, 1995.

KLUCKHOHN, Clyde. Values and value-orientations in the theory of action: An exploration in definition and classification. 1951.

LOCKE, Edwin A. The nature and causes of job satisfaction. Handbook of industrial and organizational psychology, v. 1, p. 1297-1343, 1976.

MARQUEZE, Elaine Cristina; DE CASTRO MORENO, Claudia Roberta. Satisfação no trabalho-uma breve revisão1 Job satisfaction-a short review.Política Editorial, v. 5409, n. 112, p. 69-79, 2005.

Marôco, J. Análise Estatística com o SPSS Statistics. 6. ed. Pêro Pinheiro: 2014.

O'DRISCOLL, Michael P.; BEEHR, Terry A. Moderating effects of perceived control and need for clarity on the relationship between role stressors and employee affective reactions. The Journal of social psychology, v. 140, n. 2, p. 151-159, 2000.

PORTER, Lyman W. et al. Organizational commitment, job satisfaction, and turnover among psychiatric technicians. Journal of applied psychology, v. 59, n. 5, p. 603, 1974.

BARREIROS PORTO, Juliana; TAMAYO, Alvaro. Estrutura dos Valores Pessoais: A Relação entre Valores Gerais e Laborais. Psicologia. Teoria e pesquisa, v. 23, n. 1, p. 63-70, 2007.

REGO, Arménio. Percepções de justiça: estudos de dimensionalização com professores do ensino superior. Psicologia: Teoria e Pesquisa, v. 17, n. 2, p. 119-131, 2001.

ROBBINS, S.P. Comportamento organizacional. 9 ed. São Paulo: Prentice Hall, 2002.

ROKEACH, Milton. Part I. Value systems in religion. Review of Religious Research, p. 3-23, 1969.

ROKEACH, Milton et al. The nature of human values. New York: Free press, 1973.

ROZNOWSKI, Mary; HULIN, Charles. The scientific merit of valid measures of general constructs with special reference to job satisfaction and job withdrawal. Job satisfaction: How people feel about their jobs and how it affects their performance, p. 123-163, 1992.

SCHERMERHORN JR, John R.; HUNT, James G.; OSBORN, Richard N. Fundamentos de comportamento organizacional. Bookman, 1999.

SCHWARTZ, Shalom H.; BILSKY, Wolfgang. Toward a theory of the universal content and structure of values: Extensions and cross-cultural replications. Journal of personality and social psychology, v. 58, n. 5, p. $878,1990$.

SCHWARTZ, Shalom H. Universals in the content and structure of values: Theoretical advances and empirical tests in 20 countries. Advances in experimental social psychology, v. 25, n. 1, p. 1-65, 1992.

SCHWARTZ, Shalom H. Beyond individualism/collectivism: New cultural dimensions of values. Sage Publications, Inc, 1994.

SCHWARTZ, Shalom H. Valores humanos básicos: seu contexto e estrutura intercultural. Valores e comportamento nas organizações, v. 1, p. 21-55, 2005. 
SCHWARTZ, Shalom H. A theory of cultural value orientations: Explication and applications. Comparative sociology, v. 5, n. 2, p. 137-182, 2006.

SCHWARTZ, Shalom H. Basic values: How they motivate and inhibit prosocial behavior. Prosocial motives, emotions, and behavior: The better angels of our nature, v. 14, p. 221-241, 2010.

SOARES, Cátia; AFONSO, Rosa Marina. Valores associados ao trabalho: Um estudo com a população residente na Covilhã. Análise Psicológica, v. 32, n. 1, p. 91-103, 2014.

SEAL, Frank E.; KNIGHT, Patrick A. Industrial/organizational psychology: Science and practice. Thomson Brooks/Cole Publishing Co, 1988.

SELTZER, Richard; ALONE, Sucrre; HOWARD, Gwendolyn. Police satisfaction with their jobs: Arresting officers in the District of Columbia.Police Stud.: Int'I Rev. Police Dev., v. 19, p. 25, 1996.

SPECTOR, Paul E. Job satisfaction: Application, assessment, causes, and consequences. Sage publications, 1997.

SUAR, Damodar; TEWARI, Hare R.; CHATURBEDI, Kostubh R. Subordinates' perception of leadership styles and their work behaviour.Psychology \& Developing Societies, v. 18, n. 1, p. 95114, 2006.

TAMAYO, Alvaro. Prioridades axiológicas, atividade física e estresse ocupacional. Revista de Administração Contemporânea, v. 5, n. 3, p. 127-147, 2001.

TABACHNICK, Barbara G.; FIDELL, Linda S. SAS for Windows Workbook for Tabachnick and Fidell Using Multivariate Statistics. Allyn and Bacon, 2001.

KIM, Uichol Ed et al. Individualism and collectivism: Theory, method, and applications. Sage Publications, Inc, 1994.

VERPLANKEN, Bas; HOLLAND, Rob W. Motivated decision making: effects of activation and selfcentrality of values on choices and behavior. Journal of personality and social psychology, v. 82, n. 3, p. 434, 2002.

BOAS, Luiz Henrique de Barros Vilas; DE SOUZA SETTE, Ricardo; DE BRITO, Mozar Jose. Comportamento do consumidor de produtos orgânicos: uma aplicação da teoria da cadeia de meios e fins. Organizações Rurais \& Agroindustriais, v. 8, n. 1, p. 25-39, 2006.

VROOM, V. H.. Work and Motivation. New York, NY: John Wiley \& Sons, 1964.

ZALEWSKA, Anna M. Job satisfaction and importance of work aspects related to predominant values and reactivity. International Journal of Occupational Safety and Ergonomics, v. 5, n. 4, p. 485-511, 1999.

WRIGHT, Thomas A.; CROPANZANO, Russell. Psychological well-being and job satisfaction as predictors of job performance. Journal of occupational health psychology, v. 5, n. 1, p. 84, 2000. 